\title{
Diversity in intermediate care
}

\author{
Graham P. Martin, Susan M. Peet, Graham J. Hewitt \& Hilda Parker
}

\begin{abstract}
This paper discusses the evolution of intermediate care and presents some interim observations from a survey of providers in England being conducted as part of a national evaluation of intermediate care. Telephone interviews covering various issues concerning the level of provision and style of delivery of intermediate care have been conducted with 70 services to date. Data from these are used to discuss the progress, range and nature of intermediate care in relation to clinician viewpoints and academic and official literature on the subject. Intermediate care 'on the ground' is a multiplicitous entity, with provision apparently evolving in accordance with the particularities of local need. Whilst protocols for medical involvement in intermediate care generally appear to be well established, there are some tensions concerning integration of services in a locality, care management processes and questions of flexibility and inclusiveness in relation to eligibility criteria.
\end{abstract}

Published in:

Health \& Social Care in the Community 12(2): 150-154

http://www.blackwellpublishing.com/journal.asp?ref=0966-0410

http://www.blackwell-synergy.com/links/doi/10.1111/j.0966-0410.2004.00481.x/abs/

doi:10.1111/j.0966-0410.2004.00481.x

\section{Introduction}

As a key element in the NHS Plan and the National Service Framework for Older People (Department of Health 2000; Department of Health 2001a), intermediate care is a concept which will already be familiar to those involved in the British health and social care scene. In essence, it includes services that seek to prevent inappropriate hospital admissions, facilitate supported discharges for patients who no longer need to be in hospital, and avert premature admissions to long-term care. In the last three years, central government has made a major investment into intermediate care services in England, and at the same time sought to delimit the scope and nature of intermediate care services by producing guidance specifying the kinds of interventions to which the label should apply. According to this guidance, intermediate care should be targeted at those facing unnecessarily prolonged hospital stays or inappropriate admissions to acute hospitals or long-term care, and should involve a structured intervention of active therapy over a period not usually exceeding six weeks (Department of Health, 2001b). Concerns have been raised over various aspects of the plans for intermediate care, notably questions over access to acute services and diagnostic facilities for patients, a perception that older people will simply be 'warehoused' to free acute bed space, the risk of duplicating provision or simply shifting scarce resources around a system, and the lack of an evidence base for the types of interventions enshrined in intermediate care (Grimley Evans and Tallis 2001; MacMahon 2001; Pencheon 2002; British Geriatrics Society \& Age Concern 2002).

More evidence does exist for intermediate care than is often claimed (see, for example, Steiner 2001; Parker et al. 2000; Stevenson \& Spencer 2002), but the nebulous nature of the vocabulary, combined with the relative novelty of the term 'intermediate care' itself, makes systematic literature searching difficult. There remains, however, a clear need for evaluations of services as they operate in particular local contexts. In 2001 the Department of Health (DH) Policy Research Programme with the Medical Research Council commissioned three studies to explore various aspects of provision. ${ }^{1}$ The authors of this paper are part of a team from Leicester

\footnotetext{
${ }^{1}$ See http://www.prw.le.ac.uk/intcare
} 
and Birmingham Universities carrying out one of these evaluations, looking at the costs and outcomes of intermediate care for older people in several different health and social care communities, which is due to report in 2004. Among other research activities, this study involves a survey of intermediate care provision for older people to explore the progress, range and nature of service development across England.

This paper discusses some interim observations arising from this survey in relation both to the 'policy theory' of intermediate care - in the form of guidance and advice provided by the DH and other agencies (Stevenson \& Spencer 2002; Department of Health 2001b; Department of Health 2002a)—and to clinician viewpoints on the subject, particularly concerns such as those mentioned above over the practice of intermediate care.

\section{Method}

The survey reported on here was designed to gain an overall picture of intermediate care provision across England, by collecting a variety of descriptive data on the form, function, processes and outcomes of services. These were collected on an interview schedule / pro forma, data from which were then stored in an electronic database. The pro forma was piloted and developed with services in one former Health Authority, the data from which are not included here. The questions were largely descriptive, but there was also the opportunity for respondents to mention other relevant information.

Data have so far been collected on 70 schemes out of around 120 contacted, falling largely within one former NHS region. Services and respondents were identified by contacting intermediate care co-ordinators, based in former Health Authorities or more recently in Primary Care Trusts (PCTs), who provided lists of services and team contact details for their locality. In order to obtain information which was as accurate and up-to-date as possible, the respondents interviewed were always directly involved with service provision, rather than more senior or strategic staff. The pro forma was completed for each service by conducting telephone interviews with these service representatives, generally team leaders or managers but occasionally other team members, between March and September 2002.

The analysis process for this paper focused on those questions which had a significant 'qualitative element' rather than those which required 'yes or no' or quantitative responses. These included, for example, questions on the original rationale for setting up the service, nature of and rationale for eligibility criteria, and degree of medical involvement, among others. These data were then thematically coded and analysed 'within' and 'across' services-i.e. looking to make sense of the data both by identifying common and conflicting themes from different services, and by understanding individual services coherently by linking themes within services and analysing them alongside the more factual background information collected on those services (cf. Huberman and Miles, 1998).

Due to the timing of the survey, the lists obtained of services and contacts were more accurate in some Health Authorities and PCTs than others: in the period surrounding the April 2002 strategic reorganization of the NHS, some intermediate care co-ordinators were difficult to locate, some were new in post, and some had not yet been appointed at all, leading to obvious difficulties with obtaining accurate information on individual services and resulting in the relatively low response rate to date. Coverage of the survey was therefore imperfect, although it included both rural and urban areas from across the region in question. More to the point, most of the services for which data were collected provided complete coverage of the former Health Authorities in which they were located, which meant that information was collected for the full complement of different kinds of intermediate care services within these health and social care systems.

The schemes contacted to date represent a small proportion of the totality of intermediate care provision in England and are not a random or systematic sample. Nevertheless, they are illustrative of the kinds of issues faced in planning and providing intermediate care, the complexity and heterogeneity of service models and systems, and the ways in which practitioners are looking 
This is an electronic version of an Article published in Health \& Social Care in the Community 12(2): 150-154. @ 2004 Blackwell Publishing Limited

\begin{tabular}{lr} 
& \\
Total services surveyed to date & \\
Service aim(s) (one or more per service) & 37 \\
Admission avoidance & 45 \\
Supported discharge & 45 \\
Rehabilitation & 1 \\
Not known & 9 \\
No data & \\
Residential/inpatient versus non-residential provision & \\
Services providing beds (residential/inpatient) only & 22 \\
Services providing places (home care / outpatient) only & 33 \\
Services providing both beds and places & 13 \\
Not known & 2 \\
No data & 0 \\
Funding & \\
Recurrent funding & 31 \\
Non-recurrent funding & 3 \\
Funding situation not known & 24 \\
No data & 12 \\
Involvement of health and social services & \\
Joint health and social services & 32 \\
Health only & 16 \\
Social services only & 2 \\
Not known & 3 \\
No data & 17 \\
\hline
\end{tabular}

\section{Start date of service}

Before DH guidance HSC 2001/001 (before January 2001) 38

Since DH guidance HSC 2001/001 (January 2001 or later) $\quad 7$

Not known

No data

Medical responsibility

Consultant

GP (including patient's own GP)

Consultant and/or GP

Not known

No data

\section{Number of admissions per month}

3-19

20-49

50-99

100-147

Not known

No data

Approximate average age of users

60-69

70-79

80-84

85-89

Not known

Table 1: summary information on the services contacted so far in the National Evaluation survey. In some instances no data were available from initial contact, necessitating followup calls which have yet to be carried out.

to meet the range of needs of older people in particular areas. Experiences from our evaluation 
work in other parts of the country suggest that similar issues are relevant elsewhere. We now consider a few of the themes that emerged from the data, and what these meant in practice for the intermediate care services which provided responses to the survey.

\section{Intermediate care provision cannot easily be categorized}

The 70 schemes considered here provide a divergent range of intermediate care services. Table 1 presents some background information on types of service delivery, but this does not do justice to the breadth of provision. As others have noted (Parker et al. 2000; Stevenson \& Spencer 2002; Carpenter et al. 2002), service titles do little to demystify the nature of services, with terms such as 'rapid response' or 'hospital-at-home' covering many different forms of intermediate care. Similarly, it was difficult, and potentially misleading, to fit the services as they operated in real life to the various models of intermediate care presented in the DH's (2001b) Health Service Circular. These models do not encapsulate the variety of services that were tailored to meet the particular needs of a local health and social care economy.

In meeting these needs, the functions served by ostensibly similar services varied significantly, in terms of position in the health and social care spectrum and level of intervention provided. For example residential rehabilitation services in some areas provided low-level, 'social rehabilitation' designed to promote and maintain independence among those living in the community, while in others, residential rehabilitation was an intensive intervention combining a variety of therapeutic inputs following an acute medical admission.

\section{Provision is sensitive to local service context}

The type of provision developed in a given area appears to depend to a large extent on the prevailing 'gaps' in the local system. For example, a lack of appropriate rehabilitation units following a stroke or orthopaedic admission might give rise to a need for a condition-specific intensive home-based rehabilitation service, with the extra benefits that domiciliary rehabilitation is seen to confer, while elsewhere, perceived problems with patients presenting to general practitioners (GPs) or Accident and Emergency (A\&E) with essentially 'social' needs (perhaps as simple as a breakdown of care arrangements at home) might require a low-level 'social rehabilitation' service. Thus depending on the relative stresses experienced by primary care, social services and secondary care within a locality, the character of services with similar aims may vary greatly. In this context, and perhaps more fundamentally, it is helpful to acknowledge that the nature of an 'inappropriate' admission is not fixed: rather, these events appear to depend upon (among other things) the strengths and weaknesses of a given system. 'Preventable' admissions are not always inappropriate if suitable alternatives are not in place; an 'unnecessary' admission in one system may not be unnecessary in another.

\section{Protocols for medical involvement largely well established}

For services providing care for older patients who may be suffering undiagnosed or multiple pathologies, medical involvement has been a particularly contentious issue. The early impression from the survey findings to date is that such issues are being acknowledged and addressed by many services. Those primarily therapeutic services which take referrals from professions allied to medicine, social workers and even patients themselves largely have protocols in place for assessment to identify where medical referral is needed. Admission criteria for post-acute services specify that patients require therapy, nursing or social care and not medical input.

There is also legitimate concern about patients missing out on specialist medical review where admission avoidance is an explicit aim of the intervention, especially where a GP refers directly to a service without a specialist assessment (e.g. Bulger 2002). This worry is not new: the same concerns have been expressed about early alternatives to acute admission such as hospitalat-home schemes (Shepperd \& Iliffe 2001). Some intermediate care services, however, do have access to community geriatricians, and themselves provide a direct route for comprehensive 
geriatric assessment and access to specialist care. With some services, though, arrangements for medical involvement were less clear or sometimes not yet established. Further evaluation of the extent of medical involvement in intermediate care services, especially those which aim to avoid admissions, may be of considerable utility (Wilson \& Parker 2003).

\section{Further integration of intermediate care needed}

For intermediate care to achieve its key documented aim of maximizing independence, it is evidently crucial that the care and rehabilitation of each patient is planned according to her/his needs. In some instances, the services contacted appear to be working hard towards procedures such as shared records, joint protocols and a robust screening and assessment process in order to ensure that each patient receives a bespoke plan of care. However, integration with other elements of the health and social care system varies significantly. Some services have procedures for identifying potential users from their first point of contact with the system (e.g. presentation at $A \& E$ or call to social services) and ensuring that social services, housing and the local primary care team are involved in planning discharge from an early stage. Such comprehensive mechanisms, however, are not as common as would be desirable, though plans afoot to introduce a unified assessment process (Department of Health 2002b) may expedite their diffusion.

\section{Precision versus flexibility in eligibility criteria}

On the one hand, services wish to avoid 'cherry-picking' users and to provide for all those who might benefit from their intervention even if they do not meet precise criteria; on the other hand, some services, especially those based in wards in acute hospitals, find that their beds are prone to occupation by inappropriate patients referred from overflowing acute wards. Attitudes towards this tension vary: several services stressed the importance of treating each case on its individual merits and adapting provision to meet the needs of users who, for example, might not be at immediate risk of admission to hospital, but who might enjoy increased quality of life and independence as a result of intensive rehabilitation of the sort provided. Elsewhere, though, clinicians evidently feel pressure to conform closely to intermediate care standards and are strict in their deployment of eligibility criteria, leading to a tighter screening of users and underused capacity.

\section{Intermediate care and local health and social care strategies}

Among the services contacted to date there are some striking examples of innovation in meeting the needs of service users and addressing gaps in local health and social care provision. One service, for example, was targeted specifically at those at risk of admission to long-term care following a collapse or care crisis, providing breathing space to empower users to make considered decisions about their future care arrangements. Services with devolved budgetary control were able to cater for a wide range of clients, with the power, for example, to purchase extra care to enable more dependent users than normally taken to benefit if it was felt appropriate. Several services incorporated specialist provision for specific groups, such as cognitively impaired people, while others looked to incorporate broader aspects of promoting independent living, for example through advice on entitlements and access to corollary services. Such innovative ways of realizing the principles of intermediate care may be important in contributing to the means of resolving local strains in the health and social care system, if they can promote choice, independence and empowerment for older people in a way which also addresses particular historical gaps in service provision.

\section{Discussion}

What we believe these early observations illustrate, if nothing else, is the fact that intermediate care as it is being developed and delivered is a constellation of complementary services rather than a straightforward, easily characterized model of care. It is important to appreciate that, in practice, 
it appears that intermediate care is being utilized to meet local needs of service users and gaps in provision-at least in the areas covered by this survey.

Such a finding might be viewed in two ways. On the one hand, it should provide comfort to those such as Grimley Evans and Tallis (2001), who have expressed concern that intermediate care is a top-down solution poorly suited to real-life situations, rightly pointing out the importance of local context in the success or otherwise of a systemic intervention such as intermediate care. The signs are that those responsible for implementing intermediate care are doing so in a manner sensitive to the peculiarities of local geography and existing service provision, seeking to provide a portfolio of services that plug the gaps in local care and rehabilitation to fulfil unmet need. On the other hand, such diversity could equally imply a poor adherence to those models and protocols which do exist, giving rise to poor clinical outcomes and inefficiency. What is certain is the need for careful monitoring and evaluation of the effectiveness of the different approaches to intermediate care in general, and of particular services and integrated 'single points of access' to intermediate care as they operate in their local contexts.

One risk is that a doctrinaire interpretation of intermediate care guidance might combine with the ubiquitous spectre of central targets to stifle the innovation and flexibility needed to address local need as effectively as possible. The contribution of services adhering to the spirit but not the letter of intermediate care would seem to be an important one in many health and social care communities looking to provide an effective complement of services.

The themes discussed here arise from a limited number of services and are not the result of a representative survey, but they do indicate some of the key issues arising from the development and practice of intermediate care as pursued 'on the ground'. Rather than conforming to a single ideal-type or even following a choice of predefined models, managers and practitioners of intermediate care are implementing services in a pragmatic way designed to fit into the local care system. These kinds of developments are encouraging, but of course they do not represent robust evidence of the success or otherwise of services in terms of outcomes for patients and cost-efficiency. Research must continue to build a broad evidence base sensitive to variability of services within and between localities, a challenge which is being addressed in part by the DH's programme of national intermediate care research as well as a growing body of local evaluations.

\section{Acknowledgements}

The authors would like to acknowledge the financial support for this work from the Department of Health Policy Research Programme and the Medical Research Council, and the valuable comments on earlier drafts of this paper of the other members of the Intermediate Care National Evaluation team and steering group. We would also like to thank two anonymous referees for their constructive criticism.

\section{$\underline{\text { References }}$}

British Geriatrics Society \& Age Concern (2002) Implementation of the National Service Framework and Intermediate Care seen from Geriatricians' and Older People's Perspectives. BGS / Age Concern, London

Bulger G. (2002) Intermediate care is ageist. British Medical Journal 325: 495

Carpenter I., Gladman J.R.F., Parker S.G. \& Potter J. (2002) Clinical and research challenges of intermediate care. Age and Ageing 31: 97-100

Department of Health (2000) The NHS Plan. HMSO, London

Department of Health (2001a) National Service Framework for Older People. Department of Health, London

Department of Health (2001b) Intermediate Care. Health Service Circular 2001/001 / Local Authority Circular (2001)1. Department of Health, London

Department of Health (2002a) Intermediate Care: Moving Forward. Department of Health, London 
This is an electronic version of an Article published in Health \& Social Care in the Community 12(2): 150-154. @ 2004 Blackwell Publishing Limited

Department of Health (2002b) The Single Assessment Process for Older People. Health Service Circular 2002/001 / Local Authority Circular (2002)1. Department of Health, London

Grimley Evans J. \& Tallis R.C. (2001). A new beginning for care of elderly people? Not if the psychopathology of this national service framework gets in the way. British Medical Journal 322: $807-808$

Huberman, A.M. \& M.B. Miles (1998) Data management and analysis methods. In N.K. Denzin \& Y.S. Lincoln (eds.) Collecting and Interpreting Qualitative Materials. Sage, London: 179-210

MacMahon D. (2001) Intermediate care-a challenge to specialty of geriatric medicine or its renaissance? Age and Ageing 30(S3): 19-23

Parker G., Bhakta P., Katbamna S., Lovett C., Paisley S., Parker S., Phelps K., Baker R., Jagger C., Lindesay J., Shepperdson B. and Wilson A. (2000) Best place of care for older people after acute and during subacute illness: a systematic review. Journal of Health Services Research and Policy 5(3): 176-189

Pencheon D. (2002) Intermediate care: appealing and logical, but still in need of evaluation. British Medical Journal 324: 1347-1348

Shepperd S. \& Iliffe S. (2001) Hospital at home versus in-patient hospital care (Cochrane Review). In The Cochrane Library 2001: 4. Update Software, Oxford

Steiner A. (2001) Intermediate care-a good thing? Age and Ageing 30(S3): 33-39

Stevenson J. \& Spencer L. (2002) Developing Intermediate Care: a Guide for Health and Social Care Professionals. Kings Fund, London

Wilson A. \& Parker H. (2003) Intermediate care and general practitioners: an uncertain relationship. Health and Social Care in the Community 11(2): 81-84 